\title{
A new interpretation of non-pulmonary vein substrates of the left atrium in patients with atrial fibrillation.
}

\author{
Mariano Rillo ${ }^{1}$, Zefferino Palamà ${ }^{2}$, Raffaele Punzi ${ }^{1}$, Salvatore Vitanza ${ }^{1}$, Angelo Aloisio ${ }^{1}$, \\ Silvia Polini ${ }^{1}$, Antonella Tucci ${ }^{1}$, Annalisa Pollastrelli ${ }^{3}$, Francesco Zonno ${ }^{3}$, Antonio \\ Anastasia $^{2}$, Cesare Giannattasio ${ }^{1}$, and Luigi $\mathrm{My}^{1}$ \\ ${ }^{1}$ Casa Di Cura Villa Verde \\ ${ }^{2}$ Casa di Cura Villa Verde Srl \\ ${ }^{3}$ Abbott Medical Italia SpA
}

August 7, 2020

\begin{abstract}
Background. Substrate analysis of the left atrium in patients undergoing atrial fibrillation ablation has limitations when performed by means of simple bipolar acquisition. Objective. To evaluate the incidence of low-voltages (LV) through maps constructed by means of various catheters:multipoltar (MC), omnipolar (OC) and circular catheters (CMC) with the 3D electroanatomical systems (3d-S) CARTO3 and Ensite-Precision. Methods. To assess LV we acquired maps by means of CMC and $\mathrm{MC}$ in the voltage range $0.05-0.5 \mathrm{mV}$ in 70 patients in sinus rhythm. In case of OC only, we made an intra-patient comparison of bipolar maps constructed in along, across and HD-Wave configurations by means of Ensite-Precision in the ranges of 0.05-0.5 $\mathrm{mV}$ and $0.5-1.0 \mathrm{mV}$. Basing on this comparison, we chose the range that best identified LV and characterized patchy fibrosis by analyzing a set of different colors (qualitative analysis). Finally, we performed a quantitative analysis of LV by applying the qualitative characteristics described above. Results. Basing on our settings, the optimal range for OC was 0.3-0.6 mV. OC revealed smaller LV areas than MC ( $\mathrm{p}<0.05$ or $\mathrm{p}<0.001)$, except in the lateral wall. No significant differences were observed between CMCs. The same rates of AF recurrence were found for $\mathrm{OC}$ and $\mathrm{MC}$ during the follow-up period. Conclusions.In our experience, OC does not present the limits of bipolar HD maps, though further studies are needed in order to confirm that 0.3-0.6 $\mathrm{mV}$ as $\mathrm{LV}$ optimal voltage range.
\end{abstract}

\section{INTRODUCTION}

Although pulmonary vein (PV) isolation (I) remains a cornerstone of any AF ablation (1,2,3 ), other anatomical regions of the left atrium (LA), i.e. non-PV substrates, are involved in AF, especially in persistent (Ps) AF (4,5). High density (HD) endocardial voltage mapping by means of multipolar catheters and $3 \mathrm{~d}$ electro-anatomical systems (3d-S) has been increasingly used in clinical practice to identify both left ventricular (6) and LA anatomical areas of low-voltage (LV) electrical activity $(\mathbf{7 , 8})$, which is commonly considered a marker of atrial fibrosis $(\mathbf{9})$. LA substrate modification by targeting LV zones is an ablation strategy that, in addition to PVI, tries to erase arrhythmogenic mechanisms harbored in such tissue $(\mathbf{7}, 10$ ). However, bipolar recordings have a limited ability to identify LV electrical activity, as they are subject to various influences, such as the bipole orientation expressed by the angle of attack and the activation wavefront; this can mean that electrical signals may not be recorded even when they are present $(\mathbf{1 1})$. Recent reports have described experiences of the use of new catheters with omnipolar recording capacity which do not seem to be affected by the negative influences described above with regard to bipolar HD maps $(\mathbf{1 2 , 1 3 )}$. The aim of the present study was to perform a post-hoc analysis in patients undergoing PVI, in order to evaluate the incidence of non-PV substrates detected by different diagnostic catheter technologies, including 
multipolar (MC), omnipolar (OC), and circular mapping catheters (CMC), and by means of qualitative and quantitative analysis of $\mathrm{LV}$ on applying various voltage ranges.

\section{MATERIALS AND METHODS.}

Study Population. Between February 2019 and January 2020, we enrolled 70 consecutive patients undergoing PVI for paroxysmal (P) AF ( $\mathrm{n}=40,57.14 \%)$ or PsAF $(\mathrm{n}=30,42.87 \%)$. All patients were strongly symptomatic for palpitations, fatigue, dyspnea, or chest pain, and refractory to antiarrhythmic drugs (AAD). Tab. 1 reports their baseline characteristics at the time of ablation. All patients provided written informed consent after being adequately informed of the risks and benefits. Before PVI, all patients underwent evaluation of the LA and echocardiographic measurement of the volume of the four chambers by means of the Simpson method (cut-off normal value $28 \mathrm{ml} / \mathrm{m}^{2}$ ). Exclusion criteria were: extracardiac causes of atrial fibrillation (hyperthyroidism, rheumatic diseases, electrolyte imbalance, etc), age under 18 years or over 80 years, severe valvular heart disease, presence of contraindication for oral anticoagulation, and recent percutaneous coronary intervention or cardiac surgery. After PVI, we performed HD mapping in all patients

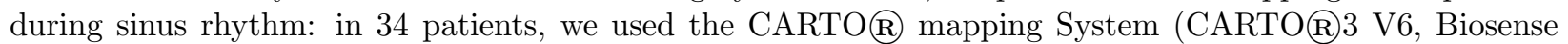
Webster, Inc., Irvine, CA, USA) and the Lasso ${ }^{\circledR}$ Nav Circular Catheter or the PentaRay@ Nav Multipolar Catheter (Biosense-Webster, Inc., Irvine, CA, USA); in 36 patients,we used the Ensite-Precision 3d system (Abbott, Minneapolis, MN) and the Inquiry AFocus II Circular Catheter or Advisor HD-Grid Mapping Catheter (Abbott, Minneapolis, MN).

Procedural Setup: Ablation Settings. PVI was carried out through contact force-guided ablation by means of a 3.5-mm open irrigated-tip catheter: SmartTouch (Biosense Webster's ThermoCool(r) SmartTouch(r), Biosense Webster, Inc., Irvine, CA, USA) or TactiCath (Abbott, Minneapolis, MN). Surface electrocardiographic leads (aVF, $\mathrm{V}_{1}$ and $\mathrm{V}_{6}$ ) and bipolar intracardiac electrograms filtered at 30 to $500 \mathrm{~Hz}$ were recorded on a Prucka-Cardiolab EP v6.9.0 Recording System (GE Healthcare,Chicago, Illinois). RF was applied point-by-point around each PV under conscious sedation, after setting ablation index values of 330 for posterior segments and 450 for anterior segments in patients in whom the SmartTouch catheter was used, and lesion index values of 5 for posterior segments, 5.5 for lateral and 6 for anterior segments in patients in whom the TactiCath catheter was used. A minimum inter-lesion distance of $6 \mathrm{~mm}$ was the endpoint in all patients, and entrance and exit block of PVs was demonstrated by applying standard pacing maneuvers and other common criteria described in several previous studies $(\mathbf{1 4 , 1 5 , 1 6 , 1 7 , 1 8 )}$.

Voltage Mapping. After PVI, the patients underwent HD mapping of the lesions around PVs and of the entire LA. All HD maps were constructed during sinus rhythm, either spontaneous or restored by electrical cardioversion. The LA anatomy was subdivided into 6 non-PV regions: anterior wall (AW), lateral wall (LW), inferior wall (IW), septal wall (SW), posterior wall (PW), and LA roof. The boundaries of each area were defined as shown in Fig.1. Any LVs of the LA were classified according to their location. In 34 patients, HD maps were acquired by means of the CMC LassoNav (17) or the MC Pentarey (17) and the Coloring/Confidense mapping algorithm of the 3d-S CARTO, which enables automated map acquisition. We performed a qualitatively analysis of each HD map acquired, based on the standard voltage range reported in the literature $(0.05-0.5 \mathrm{mV})$, to assess non-PV anatomical areas of the LA. For this purpose, we searched for numerous small islands of LV of blue/green/red color, which we considered potential patchy fibrosis, scattered within larger areas of LV>0.5 mV of purple color (healthy tissue) or within areas of LV [?]0.05 $\mathrm{mV}$ of blue/green/red color, considered dense fibrosis (DF), or of no electrical activity (only red color: scar tissue). Lesions compatible with DF or scar tissue around PVs was one of the criteria on which PVI was based. All HD maps were based on the acquisition of as many voltage points as possible, and the Tissue Proximity Indicator filter of 3d-S CARTO was used to exclude map points deemed not to be in contact with the shell. Fig. $1 \mathrm{~A}, \mathrm{C}$ and $\mathrm{E}$ show HD maps acquired by means of 3d-S CARTO. In 36 patients, HD maps were acquired by means of the CMC AFocus (18) or a 16-electrode HD configuration with $3 \mathrm{~mm}$ spacing by means of the standard Advisor OC HD-Grid (18). For both types of catheter, the best duplicate algorithm of 3d-S Ensite Precision was used; this creates voltage maps by using the electrogram with the highest amplitude in the same position when multiple points are projected, while the worst voltage signals 
are automatically discarded, each time the catheter passes a point. We evaluated tissue contact by using the Proximity Indicator of the 3d-S Ensite Precision and exploiting the flexibility of the OC, which folds when it comes into contact with the wall. The voltage range used to characterize the substrate with the CMC AFocus was $0.05-0.5 \mathrm{mV}$. The same range was adopted when the CMC LassoNav and MC Pentarey were used. Qualitative analysis was performed in the same way as for the maps acquired with 3d-S CARTO. Only in the case of the OC did we try to identify the optimal voltage range for the definition of non-PV substrates. To do so, in each patient with LV in the maps constructed by means of the OC (11/18 patients), we compared the bipolar maps in the "along" and "across" configuration of the Ensite Precision with the omnipolar maps in the HD-wave configuration; in this operation, we used two contiguous voltage ranges: $0.05-0.5 \mathrm{mV}$ and 0.5-1 mV. Indeed, the 3D-S Ensite-Precision allows us to change the voltage range even after completing and saving the maps and the patient has left the electrophysiology laboratory, and subsequently to compare the new maps. We used a total of $33 \mathrm{HD}$-wave and bipolar maps for the intra-patient comparison in each range considered. Anatomical areas of gray color were considered to be scar tissue and those of purple color to be normal tissue. On the basis of the results of the comparison of the 2 voltage ranges, we chose the range more able to identify anatomical areas that were characterized by a set of green/red/yellow/blue/gray/purple colors, which we considered compatible with patchy fibrosis. Subsequently, we considered the voltage value of 1 point of purple color and 1 point of gray or red color, close to the set of different colors, and at least 5 points of purple color and 5 points of gray or red color inside the set of different colors, in order to extrapolate mean, minimum and maximum values able to identify the new range to be used for further evaluations. Scar tissue, as indicated by the gray color around the PV lesion, was one criterion of PVI. All HD maps were based on the acquisition of as many voltage points as possible; we evaluated tissue contact by using the Proximity Indicator of the Ensite Precision and exploiting the flexibility of the OC, which folds when it comes into contact with the wall. Finally, in all patients with non-PV LV identified by means of both 3d-S, and with qualitative characteristics of HD maps compatible with potential patchy fibrosis, we also tried to quantify the extent of marked (M) LV areas as a percentage of the surface area of the 6 different LA anatomical regions; these were calculated in square centimeters from the measurements yielded by the two 3d-S. Fig. 1 B, D and F show the HD maps acquired by means of Ensite Precision. Omnipolar signals were occasionally compared with the bipolar signals acquired through the distal dipole of the ablation catheter positioned in contact with the electrodes of the HD-Grid detecting electrical activity. However, these comparisons were not used for evaluations in this study. Fig. 2 shows an example of direct comparison.

Post-ablation management and follow-up. The patients were followed up for a period of about 12 months. The protocol included the prescription of AAD: for a 3-month blanking period in patients with PAF and without interruption in patients with PsAF. Patients underwent clinical follow-up examinations 3, 6, 9 and 12 months after the procedure. AAD was resumed in patients with PAF in the event of recurrence. A 24-h Holter recording was performed at the scheduled controls. Anticoagulant therapy was continued indefinitely on the basis of the CHADSvasc score ([?]2 in men and [?]3 in women).

Statistical Analysis. Continuous variables are expressed as mean+-SD, and categorical variables as percentages. The Student t test and Pearson's chi-square test were used to compare continuous and dichotomous variables, respectively. We constructed Kaplan-Meier curves to illustrate 1-year freedom from AF recurrence, using a log-rank test. Two-tailed tests were considered statistically significant at the level of 0.05 . All analyses were performed by means of STATA 13.1 (STATA Corp., Texas, US).

\section{RESULTS.}

Patients. The two groups of patients(with PAF and PersAF) were homogeneous in terms of age, sex, diabetes, coronary artery disease and body mass index (Tab. 1). Hypertension and LA dilation were more frequent in PsAF than in PAF patients $(\mathrm{p}=0.04$ and $\mathrm{p}<0.001$ respectively), while left ventricular ejection fraction $>50 \%$ and normal LA were more frequent in PAF than in PsAF $(\mathrm{p}=0.01$ and $\mathrm{p}<0.001$ respectively - Tab. 1).

Procedural data. Complete PVI with bidirectional conduction block was achieved in all 70 patients by means of pacing maneuvers and HD mapping. The procedural time, including HD mapping, was $126+-$ 
13 minutes in patients who underwent PVI and mapping by CMC LassoNav or MC (p>0.5), and $119+-$ 15 minutes in patients in whom CMC AFocus or OC ( $>0.5)$ was used for mapping. The numbers of voltage points acquired were:1,583+-431 with CMC AFocus versus 1,501+-317 with LassoNav ( $>0.5)$, and $18,626+-4,617$ with $\mathrm{OC}$ versus $2,037+-343$ with MC $(\mathrm{p}<0.0001)$. In our intra-patient comparison of "along" and "across" bipolar maps, we observed both an increase in LV on passing from the 0.05-0.5 $\mathrm{mV}$ range to the 0.5-1 mV range and a shift from the set of different colors to gray (transformation of areas characterized by potential patchy fibrosis into scar) in all 11 patients re-evaluated (100\%). Comparison of the 22 omnipolar maps (HD-wave maps) also showed an increase in LV in 100\% of patients re-evaluated when the 0.5-1.0 $\mathrm{mV}$ range was used, but with a shift from purple to the set of different colors (transformation of areas characterized by normal tissue into potential patchy fibrosis). An example of these comparisons is shown in Fig. 3. HD-wave maps in the $0.5-1.0 \mathrm{mV}$ range were therefore used to analyze the voltage value of 1 point of purple color and 1 point of gray or red color close to and inside the set of different colors. The lower voltage cut-off was a mean value of $0.3+-0.05 \mathrm{mV}$, while the higher voltage cut-off was $0.6+-0.03 \mathrm{mV}$ (Fig.4). Onthe basis of these results, we defined the optimal voltage range, which was between 0.3 and 0.6 $\mathrm{mV}$, and used this value in our further evaluations. After ablation, mild pericardial effusion occurred in 3 of the 70 patients $(4.3 \%)$; this only required observation for two additional days of hospitalization and echocardiographic evaluation.

Comparison of patients with and without non-PV LV. Table 2 shows our comparison of patients with and without non-PV LV. LVs were found in 38 of 70 patients (54.3\%). From the comparison between HD maps, no statistically significant differences emerged, in terms of the number of patients with LV areas, between CMC AFocus and CMC LassoNav $(\mathrm{p}=0.725)$, or between OC and MC $(\mathrm{p}=0.332)$. No LV were found in 32 of 70 patients (45.7\%), nor did any statistically significant differences emerge, in terms of patients without LV areas, between CMC AFocus and CMC LassoNav ( $\mathrm{p}=0.725)$, or between OC and MC $(\mathrm{p}=0.332)$.

Comparison of marked LV areas: CMC AFocus versus LassoNav and OC versus MC. Asno LVs were found in the LA roof in any patient, this anatomical area was excluded from the analysis. Fig. 5 reports the mean values of LV percentage in each LA anatomical area analyzed (MLV). No significant differences were found between the MLV areas identified by the 2 CMC, while a lower percentage of LV was identified by $\mathrm{OC}$ than by MC in all the anatomical areas analyzed, except for the LW (Fig. 5).

Clinical outcomes. After the blanking period, 18/70 (25.7\%) of all patients had recurrences, without significant differences between patients with HD maps acquired by means of the two CMC (AFocus versus LassoNav, $\mathrm{p}=0.9)$ nor between $\mathrm{OC}$ and $\mathrm{MC}(\mathrm{p}=0.8)$. Fig. 6 shows the Kaplan-Meier curves of time to the first $\mathrm{AF}$ recurrence; no significant differences were observed in terms of freedom from AF episodes during follow-up in all patients (AFocus versus LassoNav, $\mathrm{p}=0.6$ and $\mathrm{OC}$ versus $\mathrm{MC}, \mathrm{p}=0.8$ ).

\section{DISCUSSION.}

In several recent studies, LA bipolar endocardial voltage maps constructed by acquiring thousands of voltage points by means of 3d-S (19) have emerged as a tool for defining AF substrates during RF ablation procedures (20 ). Indeed, LV has been considered a surrogate marker of the presence of atrial fibrosis, and may play a role in giving rise to the mechanisms underlying AF, especially in the case of PsAF $(\mathbf{2 1 , 2 2})$. In these studies, however, mapping strategies and definitions of LV areas were heterogeneous. The electrical signals recorded by catheters are converted by $3 \mathrm{~d}-\mathrm{S}$ into color-coded voltage maps. These maps, however, may vary according to the catheters and $3 \mathrm{~d}$ systems used. The problem is that several non-substrate factors can theoretically influence electrogram voltage $(\mathbf{2 3 , 2 4})$.Activation direction, electrode spacing, electrode size, filter settings, point density and tissue contact are all factors that potentially influence HD maps; the technical challenges raised by these factors were discussed in a very interesting paper by Sim et al. (25 ). In particular, the relationship between the orientation of the recording bipole and the wave-front bipole may influence the arrival time of the activating wave-front at each electrode. A solution to this problem may be provided by a new mapping technique, known as omnipolar mapping; this is based on the use of a $3 \mathrm{~d}$ mapping system and a multi-electrode catheter that allows simultaneous recordings of unipolar electrograms, and which spans $2 \mathrm{~d}$ 
and 3d space to derive conduction velocity and wave-front direction (26 ). Omnipolar mapping therefore enables detailed characterization of myocardial activation in a way that is insensitive to catheter orientation; thus, the use of OC and -Ensite-Precision may be able to improve the detection of abnormal atrial substrate by using electrogram amplitude characteristics. Identifying LV areas also depends on the recording window specified for analysis and on the related voltage thresholds chosen for the definition of LV, since these factors can cause HD maps to vary markedly. Data on the relationships between pre-ablation atrial fibrosis and atrial voltage thresholds are not currently available, and no true voltage threshold for atrial anomalies has been established. However, most studies have used a voltage amplitude $<0.05 \mathrm{mV}$ to identify scar areas and a value of $>0.5 \mathrm{mV}$ to identify normal tissues, while the range between 0.05 and $0.5 \mathrm{mV}$ is commonly used to define the LV that identifies the presence of underlying anomalies in the atrial structure. In our study, we chose the 0.05-0.5 mV range adopted in several studies that have used CARTO and Ensite-Precision, in order to construct HD maps through the recording of electrical signals acquired from the CMC LassoNav, CMC AFocus or MC. For HD maps acquired by means of Ensite-Precision and OC, we identified a range of LV that was more suited to representing atrial fibrosis. This was because Ensite-Precision and OC differ from CARTO and MC in terms of their ability to select the bipolar electrograms. Indeed, by comparing different orientations of bipolar electrograms from the OC electrode, omnipolar electrograms acquired by means the same catheter match those of the largest bipolar electrogram, thereby eliminating the influence of reduced amplitude due to activation direction $(\mathbf{1 1 , 1 3})$. This relative increase in electrogram amplitude has been shown to change the voltage threshold by which tissue can be histologically defined as scar. This concept was demonstrated by a recent study on ventricular myocardial voltage, which reported that, when performing omnipolar mapping, adopting a scar threshold of $1.5 \mathrm{mV}$ (a value far higher than the standard $0.5 \mathrm{mV}$ for conventional bipolar mapping) corresponded better to electrophysiologist-determined scar than the area determined from bipolar signals $(\mathbf{2 7})$. Additionally, Takigawa et al. explored the optimal threshold in 2 configurations of OC in 6 infarcted sheep, and compared the impact of electrode spacing and bipolar direction on the scar threshold $(\mathbf{2 8})$. They found that, although scar areas were well distinguished from areas of healthy tissues in any bipolar configurations with OC, bipolar spacing of $1 \mathrm{~mm}$ showed relatively lower accuracy, and bipolar voltages generally increased as the inter-electrode spacing increased in both healthy and scar areas. It has also been shown that LV areas documented by OC and HD-wave configuration during VT ablation procedures are smaller than the same areas identified by means of the "along" and "across" bipolar configuration (29). Substrates in the LA of patients with AF are probably similar to, but not the same as, scar tissue due to infarction of the ventricular myocardium. It has been suggested that re-entry mechanisms do not occur in densely fibrotic areas or scar tissue because there are not enough cardiomyocytes to allow impulse propagation $(\mathbf{3 0})$, nor does it occur in mostly normal tissue, because any reentry circuits that are established are unstable and self-terminate promptly. We identified anatomical areas characterized by a set of different colors (green/red/yellow/blue/gray/purple). To do so, we used the best duplicate algorithm of Ensite-Precision, after setting an optimal voltage range, and the OC of 16 electrodes with $3 \mathrm{~mm}$ inter-electrode spacing, which is the optimal spacing according to the results of the study by Takigawa. In our view, this approach offers a simple method of searching for potential and selective electroanatomical substrates underling AF, and probably constitutes the basis of truly tailored ablation. Indeed, our approach, which was both qualitative and quantitative, helped us to identify potential patchy fibrosis; thus, it was probably able to define a substrate that could represent local non-PV conductive alterations that influence anisotropy. This substrate probably plays a critical role in giving rise to AF mechanisms, as described by some authors $(\mathbf{3 1 , 3 2})$. We used the OC to search for the range that could best identify this substrate. This revealed that increasing the range from $0.05-0.5 \mathrm{mV}$ to $0.5-1 \mathrm{mV}$ increased the possibility of observing electrical alterations compatible with patchy fibrosis. By contrast, if bipolar signals are used, this capacity is lost, as scar tissue is more evident, and this probably does not express true electrical alterations. The optimal range of $0.3-0.6 \mathrm{mV}$ that we identified characterized the non-PV substrate more selectively than the range reported in the literature. Finally, the results from our quantitative analysis of the substrate identified by means of $\mathrm{OC}$ and the optimal range were similar to those reported by Masuda et al. (33 ). These authors compared "along" and "across" CMC bipolar HD maps with OC omnipolar HD maps, and found a significantly lower percentage of LV areas in the majority of LA anatomical areas studied by means 
of OC than in those evaluated by means of bipolar HD maps. In our experience, the total number of voltage points acquired was significantly higher with $\mathrm{OC}$ than with $\mathrm{MC}$, thus allowing us to construct maps with higher density. These findings are also in line with those of Masuda. Moreover, the procedural time needed for the acquisition of voltage points was similar among the different catheters used; this was probably due to the fact that more complex mapping is required in patients with PsAF, in whom OC were preferentially used, while CMC mapping yielded fewer voltage point.

Limitations. This study has some limitations. Firstly, it was single-center and retrospective; future studies should be prospective and multi-center and include direct comparison of diagnostic catheter technologies in a randomized setting. Moreover, only a small number of patients were included, while larger series are needed in order to fully assess the efficacy of $0.3 \mathrm{mV}$ as the lower and $0.6 \mathrm{mV}$ as the upper cut-off limits when OC are used to identify substrates that may play a role in maintaining AF. In addition, our search for LV was arbitrarily performed only during sinus rhythm and no comparison was made during AF; the superiority of the sinus rhythm approach therefore remains to be validated. Finally, we did not evaluate the impact,in terms of outcome, of tailored RF ablation of the substrates identified by means of OC in comparison with a matched control group of patients in whom the substrates were identified by means of MC.

\section{CONCLUSION.}

According to our experience, the combined use of OC and Ensite-Precision selectively identifies substrates that are potentially responsible for the mechanisms of AF by detecting LV that are not affected by the orientation of the catheter with respect to the activating wave-front. This approach therefore overcomes one of the limitations of using HD maps derived from bipolar catheters. In our study, the 0.3-0.6 mV range associated with a qualitative analysis identified potential fibrotic substrates that could play a role in the mechanism of sustained AF better than the standard range of $0.05-0.5 \mathrm{mV}$. However, further studies are needed in order to determine whether $0.3-0.6 \mathrm{mV}$ is the optimal range within which to identify $\mathrm{LV}$ areas by means of OC and Ensite-Precision.

\section{Bibliography}

1. Haissaguerre M, Jais P, Shah D.C. et al. Spontaneous initiation of atrial fibrillation by ectopic beats originating in the pulmonary veins. $\mathrm{N}$ Engl J Med 1998. https://www.nejm.org/doi/full/10.1056/NEJM199809033391003

2. Shiroshita-Takeshita A, Brundel BJ, Nattel S. Atrial fibrillation: basic mechanisms, remodeling and triggers. J Interv Card Electrophysiol 2005. https://link.springer.com/article/10.1007/ s10840-005-2362-y

3. Calkins H, Hindricks G, Cappato R et al (2017). HRS/EHRA/ECAS/APHRS/SOLAECE expert consensus statement on catheter and surgical ablation of atrial fibrillation. Heart Rhythm http://dx.doi.org/10.1016/j.hrthm.2017.05.012

4. H. Lau D, Linz D, Schotten U, Mahajan R, Sanders P, Kalman J.M. (2017). Pathophysiology of paroxysmal and persistent atrial fibrillation: rotors, foci and fibrosis Heart Lung Circulhttps://www.ncbi.nlm.nih.gov/pubmed/28610723

5. Marrouche NF, Wilber D, Hindricks G et al (2014). Association of atrial tissue fibrosis identified by delayed enhancement MRI and atrial fibrillation catheter ablation: the DECAAF study. JAMA. https://www.ncbi.nlm.nih.gov/pubmed/24496537

6. Tschabrunn CM, Roujol S, Dorman NC, Nezafat R, Josephson ME, Anter E. (2016). High-resolution mapping of ventricular scar: comparison between single and multielectrode catheters. CircArrhythmElectrophysiol. https://www.ahajournals.org/doi/abs/10.1161/circep.115.003841

7. Rolf, S., Kircher, S., Arya et al. (2014). Tailored atrial substrate modification based on low-voltage areas in catheter ablation of atrial fibrillation. Circ ArrhythmElectrophysiol. https://www.ahajournals.org/doi/full/10.1161/circep.113.001251

8. Jadidi A.S., Lehrmann H, MD, Keyl C et al (2016). Ablation of persistent atrial fibrillation targeting low-voltage areas with selective activation characteristics. CircArrhythmElectrophysiol https://www.ahajournals.org/doi/full/10.1161/CIRCEP.115.002962 
9. Kottkamp, H. Human atrial fibrillation substrate: Towards a specific fibrotic atrial cardiomyopathy. Eur Heart J. 2013;2731-8. https://doi.org/10.1093/eurheartj/eht194

10. Kircher S, Arya A, Altmann D et al (2017). Individually tailored vs. standardized substrate modification during radiofrequency catheter ablation for atrial fibrillation: a randomized study. EP Europace. https://doi.org/10.1093/europace/eux310

11. Haldar S.K, Magtibay K, Porta-Sanchez A et al (2017). Resolvingbipolarelectrogram voltages during atrial fibrillation usingomnipolar mapping. CircArrhythmElectrophysiol. https://www.ncbi.nlm.nih.gov/pubmed/28887362

12. Yamagushi T, Fukui A, Node K. (2019). Bipolar voltage mapping for the evaluation of atrial substrate: can we overcome the challenge of directionality? J Atr Fibrillation. https://www.ncbi.nlm.nih.gov/pmc/articles/PMC6533827

13. Masse S, Magtibay K, Jackson N, Asta J, Kusha M, Zhang B, Balachandran R, Radisic M, Deno D, Nanthakumar K. Resolving myocardial activation with novel omnipolarelectrograms. CircArrhythmElectrophysiol. 2016;9:e004107. doi: 10.1161/CIRCEP.116.004107

14. Haissaguerre M, Jais P, Shah DC, Hocini M, Yamane T, Disenhofer I, Chauvin M, Garrigue S, Clementy J. Electrophyiological breakthroughs from the left atrium to the pulmonary veins.Circulation. 2000;102:2463-2465 doi: 10.1161/01.CIR.102.20.2463

15. Squara F, Frankel DS, Schaller R, Kapa S, Chik WW, Callans DJ, Marchlinski FE, Dixit S. Voltage mapping for delineating inexcitable dense scar in patients undergoing atrial fibrillation ablation: a new end point for enhancing pulmonary vein isolation. Heart Rhythm. 2014;11:1904-1911. doi: 10.1016/j.hrthm.2014.07.027.

16. Huo Y, Kronborg MB, Richter U et al (2020). Electrophysiological findings during atrial fibrillation reablation: extending from pulmonary vein reconnection to sequential bipolar voltage map information. J Cardiovasc Electrophysiolhttps://www.ncbi.nlm.nih.gov/pubmed/32037614

17. Kim J, Desai S, Jadonath R, Beldner SJ. The importance of bidiretional block during pulmonary vein isolation. Pacing Clin Electrophysiol 2013; 36(5):e143-5 (ISSN: 1540-8159).

18. Squara F, Liuba I, Chik W, Santangeli P, Zado ES, Callans DJ, Marchlinski FE. Loss of local capture of the pulmonary vein myocardium after antral isolation: prevalence and clinical significance. $\mathrm{J}$ Cardiovasc Electrophysiol 2015 Mar;26(3):242-50. doi: 10.1111/jce.12585.

19. Kapa S, Desjardins B, Callans DJ, Marchlinski FE, Dixit S. Contact electroanatomic mapping derived voltage criteria for characterizing left atrial scar in patients undergoing ablation for atrial fibrillation. J Cardiovasc Electrophysiol. 2014;25:1044-1052. doi: 10.1111/jce.12452.

20. Andronache M, Drca N, Viola G. (2019). High-resolution mapping in patients with persistent AF. AER Journal https://doi.org/10.15420/aer.2018.57.1

21. Tzeis S, Asvestas D, Vardas P. (2019). Atrial fibrosis: traslational considerations for the management of AF patients. AER Journal https://www.aerjournal.com/articles/atrial-fibrosis-translational

22. Hunter R.J, Liu Y, Lu Y, Wang W, Schilling J.R. (2012). Left atrial wall stress distribution and its relationship to electrophysiologic remodeling in persistent atrial fibrillation. Circulation: Arrhythmia and Electrophysiology. https://www.ahajournals.org/doi/abs/10.1161/circep.111.965541

23. Josephson ME, Anter E. (2015). Substrate mapping for ventricular tachycardia. Assumptions and misconceptions. JACC Clin Electrophysiol. https://www.ncbi.nlm.nih.gov/pubmed/29759461

24. Anter E, Tschabrunn CM, Josephson ME. (2015). High-resolution mapping of scar-related atrial arrhythmias using smaller electrodes with closer interelectrode spacing. CircArrhythmElectrophysiol. https://www.ncbi.nlm.nih.gov/pubmed/25792508

25. Sim I, BishopM, O'NeillM and E. Williams S.(2019). Left atrial voltage mapping: defining and targeting the atrial fibrillation substrate. Journal of Interventional Cardiac Electrophysiology. https://link.springer.com/article/10.1007/s10840-019-00537-8

26. Deno DC, Balachandran R, Morgan D, Ahmad F, Masse S, Nanthakumar K. (2017). Orientationindependent catheter-based characterization of myocardial activation. IEEE Trans Biomed Eng. https://www.ncbi.nlm.nih.gov/pubmed/27411215

27. Magtibay K, Masse S, Asta J et al.(2017). Physiological assessment of ventricular myocardial voltage 
using omnipolar electrograms. J Am Heart Assoc. https://www.ncbi.nlm.nih.gov/pubmed/28862942

28. Takigawa M, Relan J, Kitamura $\mathrm{T}$ et al. (2019). Impact of spacing and orientation on the scar threshold with a high-density Grid catheter. CircArrhythmElectrophysiol2019;https://www.ahajournals.org/doi/10.1161/CIRCEP.119.007158

29. Okubo K, Frontera A, Bisceglia C et al (2019). Grid mapping catheter for ventriculartachycardia ablation. CircArrhythmElectrophysiol. https://www.ahajournals.org/doi/10.1161/CIRCEP.119.007500

30. Vigmond E, Pashaei A, Amraoui S, Cochet H, Haissaguerre M. (2016). Percolation as a mechanism to explain atrial fractionated electrograms and reentry in a fibrosis model based on imaging data. Heart Rhythm https://www.ncbi.nlm.nih.gov/pubmed/26976038

31. Nattel S. (2017). Molecular and cellular mechanisms of atrial fibrosis in atrial fibrillation. JACC: Clinical Electrophysiology https://electrophysiology.onlinejacc.org/content/3/5/425

32. Zahid S, Boyle P.M., Schwarz E.L. et al. (2016). Patient-derived models link re-entrant driver location in atrial fibrillation to fibrosis spatial pattern. Cardiovascular Research https://academic.oup.com/cardiovascres/article/110/3/443/1744836

33. Masuda M, Asai M, Iida O et al.(2019). Left atrial voltage mapping with a direction-independent grid catheter: comparison with a conventional circular mapping catheter. $J$ Cardiovasc Electrophysiolhttps://www.ncbi.nlm.nih.gov/pubmed/31701587

Table 1 - Baseline characteristics of patients at the time of ablation

\section{Row Labels}

\section{All Patients}

PAF

PsAF

\section{PAF versus PsAF}

$p$ value

$(\mathrm{n}=70)$

$(\mathrm{n}=40)$

$(\mathrm{n}=30)$

Age (SD)

$64.5+-8.9$

$64,5+-9$

$64+-9$

$\mathrm{p}=0.20$

Male

$47(67.1 \%)$

$25(62.5 \%)$

$22(73.3 \%)$

Hypertension

$58(82.8 \%)$

$30(75 \%)$

$28(93.3 \%)$ 
$\mathrm{p}=0.04$

Diabetes

$14(20 \%)$

$5(12.5 \%)$

$9(30 \%)$

$\mathrm{p}=0.07$

Obese (bmi [?]30)

$18(25.7 \%)$

7 (17.5\%)

$11(36.7 \%)$

$\mathrm{p}=0.47$

Overweight (bmi [?]25)

$45(64.3 \%)$

$28(70 \%)$

$17(56.7 \%)$

$\mathrm{p}=0.19$

CAD

$10(14.3 \%)$

$6(60 \%)$

$4(40 \%)$

$\mathrm{p}>0.5$

Type of AF

$40(57.2 \%)$

$30(42.8 \%)$

AF duration

[?]48 m

$27(38.6 \%)$

$14(35 \%)$

$13(43.3 \%)$

$\mathrm{p}=0.07$

[?]36 m

$40(57.1 \%)$

$23(57.5 \%)$

$17(43.5 \%)$ 
$\mathrm{p}=0.72$

LVEF [?] $30 \%$

$8(11.43 \%)$

$4(50 \%)$

$4(50 \%)$

$\mathrm{p}=0.07$

LVEF [?] $50 \%$

$62(88.57 \%)$

$36(58 \%)$

$26(42 \%)$

$\mathrm{p}=0.01$

LA volume $\left(\mathrm{ml} / \mathrm{m}^{2}\right)$

[?]28

$37(53 \%)$

$13(35 \%)$

$24(65 \%)$

$\mathrm{p}<0.0001$

$<28$

$33(47 \%)$

$27(82 \%)$

$6(18 \%)$

$\mathrm{p}<0.0001$

Values are expressed as numbers and percentages. AF=Atrial Fibrillation; bmi=body mass index; bsa=body surface area; $L A=$ Left Atrium , LVEF=Left Ventricular Ejection Fraction; $m=$ months; mm=millimeters; $\mathrm{ml} / \mathrm{m}^{2}=$ milliliters per square meter; PAF=paroxysmal AF; PsAF= persistent AF; SD Standard Deviation.

Table 2 - Patients with and without non-PVareas of low voltage

\begin{tabular}{llllllll}
\hline Patients & Tot. N. & AFocus & Lasso & p & HDGrid & Pentaray & p \\
\hline All patients & 70 & $18(25.7 \%)$ & $17(24.3 \%)$ & & $18(25.7 \%)$ & $17(24.3 \%)$ & \\
LV & $38(54.3 \%)$ & $5(13.3 \%)$ & $8(21 \%)$ & 0.73 & $11(28.9 \%)$ & $14(36.8 \%)$ & 0.33 \\
no LV & $32(45.7 \%)$ & $13(40.6 \%)$ & $9(28.1 \%)$ & 0.24 & $7(21.9 \%)$ & $3(9.4 \%)$ & 0.16 \\
\hline
\end{tabular}

Comparison of CMC AFocus versus CMC LassoNav and of OC HD-Grid versus MC Pentarey. Values are expressed as numbers and percentages. $L V=$ low voltage; no $L V=$ no low voltage

\section{FIGURES}

Fig. 1. Qualitative and quantitative analysis of LV: HD maps acquired by means of OC and MC. 


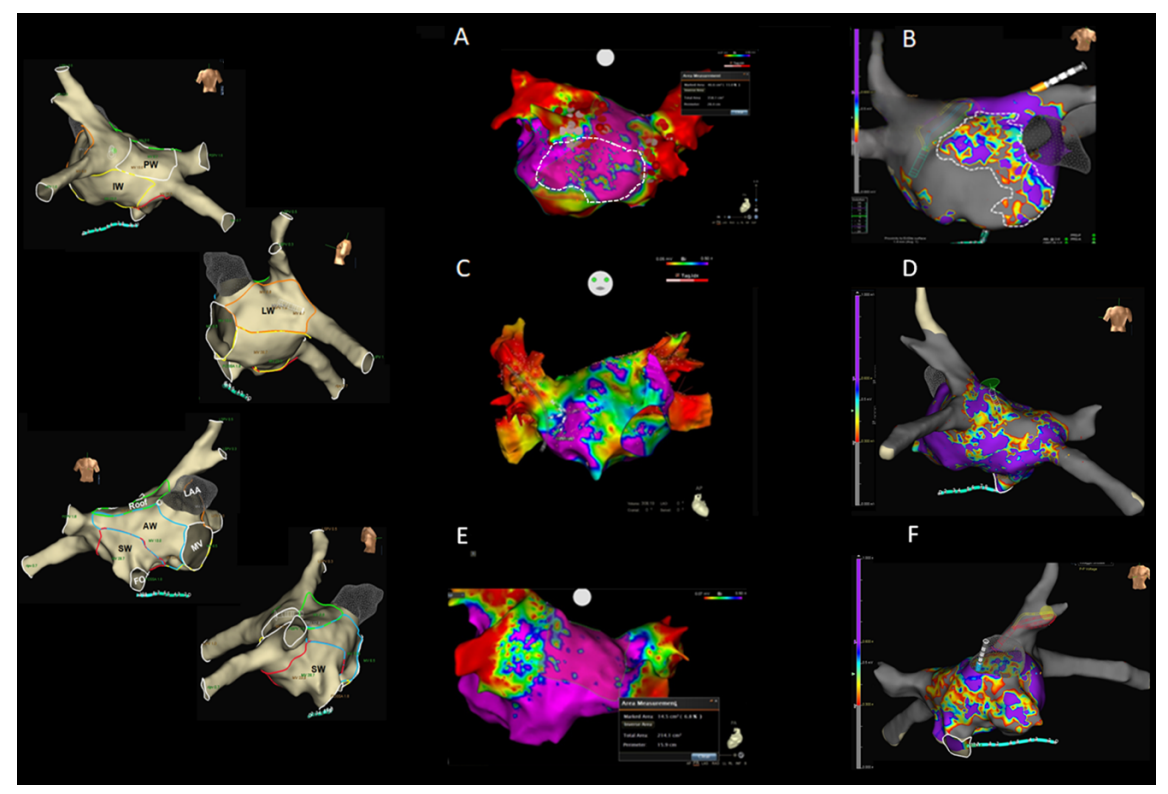

Left panel: the anatomical areas acquired with the $3 \mathrm{~d}$ electro-anatomical systems (3d-S). Right panel:A, C and $\mathrm{E}$ show the maps acquired by means of the multipolar catheter and 3d-S CARTO 3, while B, D and F show the maps acquired by means of the OC and 3d-S Ensite Precision. A.PA projection. PsAF and dilated LA. Map with 2,520 points acquired in the $0.05-0.5 \mathrm{mV}$ voltage range. The IW presents $\mathrm{LV}$, characterized by small islands of blue/green/redcolors (potential patchy fibrosis) within a larger area of healthy tissue (purple). According to our results, this map could be influenced by the limitations of bipolar recordings.B. AP Projection. PsAF and dilated LA. Map with 28,000 points acquired in the $0.3-0.6 \mathrm{mV}$ voltage range. The entire AW presents LV. A large area of different colors (dashed area) is clearly visible; this is characterized by small islands of normal tissue (purple), separated by probable patchy fibrosis (blue/green/yellow/red) inside the remaining AW; this latter area displays no electrical activity (gray) and is unable to mediate re-entry. C. AP projection. PsAF and non-dilated LA. The entire AW presents LV and is green in color (compatible with dense fibrosis) with only some small islands of healthy tissue (purple) and scar tissue (red), which poorly represent patchy fibrosis. From our point of view, this substrate is unlikely to be able to mediate re-entry. However, this map could also be influenced by the limitations of bipolar recordings D. PA Projection. Paroxysmal AF and non-dilated LA. The PW presents LV that is qualitatively compatible with patchy fibrosis. E. PA Projection. PsAF and dilated LA. The IW presents normal tissue, and the PW a few small islands of LV which are not able to mediate re-entry. F. AP Projection. The same patient as in D. The map shows a prevalence of green and red colors that are more compatible with dense fibrosis and less so with patchy fibrosis, indicating a substrate that is probably unable to mediate re-entry.

$A W=$ anterior wall, IW=inferior wall, LA=left atrium, $L V=l o w$ voltage, OC= Omnipolar Catheter, PsAF=persistent atrial fibrillation, $P W=$ posterior wall.

Fig. 2 Direct comparison of bipolar versus omnipolar recordings. 


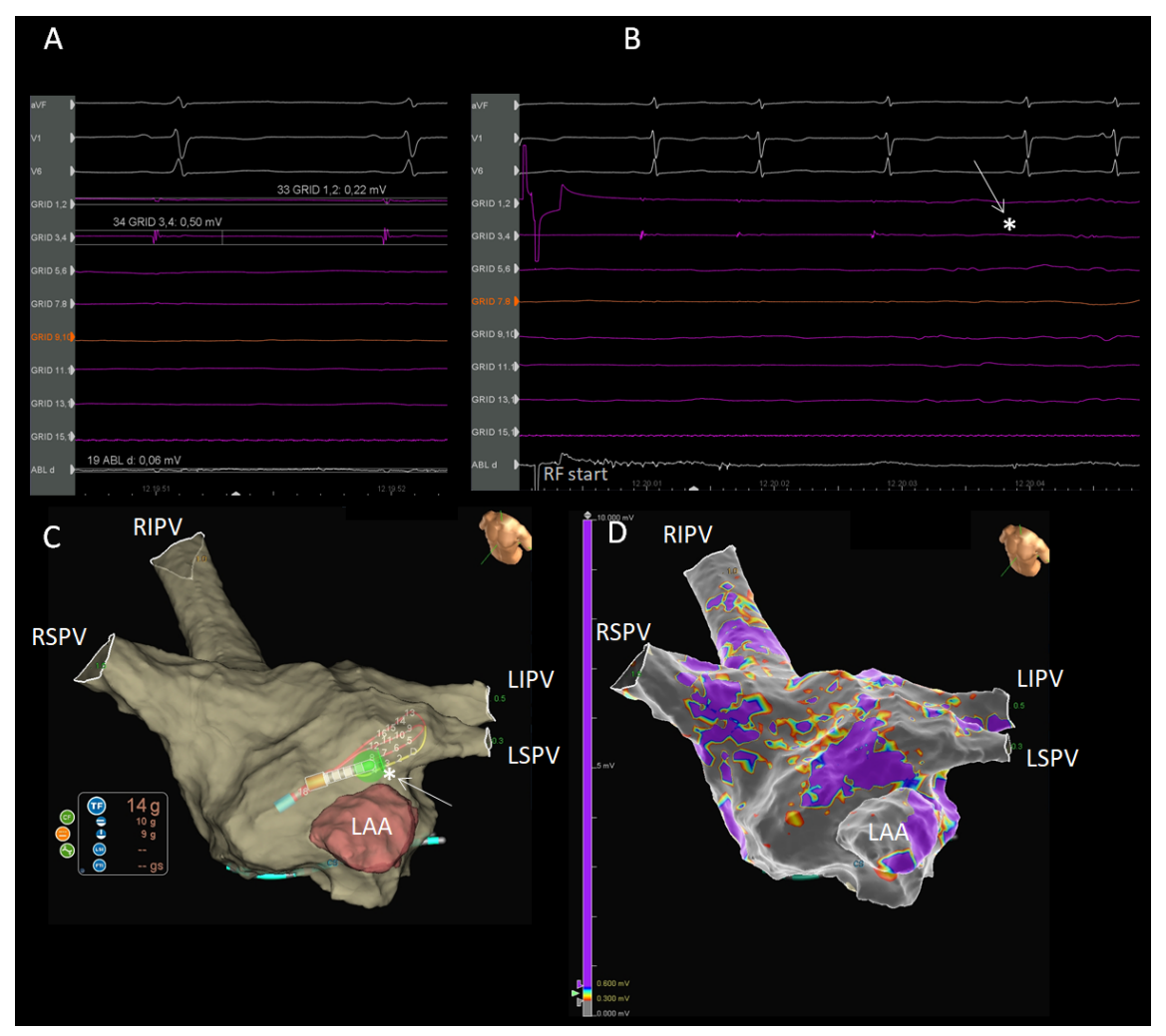

A. Pair 3,4 of the HD-Grid records a $0.5 \mathrm{mV}$ signal, while the signal recorded by pair 1,2 is of $0.22 \mathrm{mV}$. The distal dipole of the ablation catheter records a signal of $0.06 \mathrm{mV}$. B. Ablation catheter contact force of 14 grams during PVI. Complete disappearance of the HD-Grid signal at the fourth beat after the beginning of RF (white arrow and star). $\mathbf{C}$ and $\mathbf{D}$. The related $3 \mathrm{~d}$ anatomical maps.

Fig. 3. Intra-patient HD map: comparison of along, across and HD-wave maps 


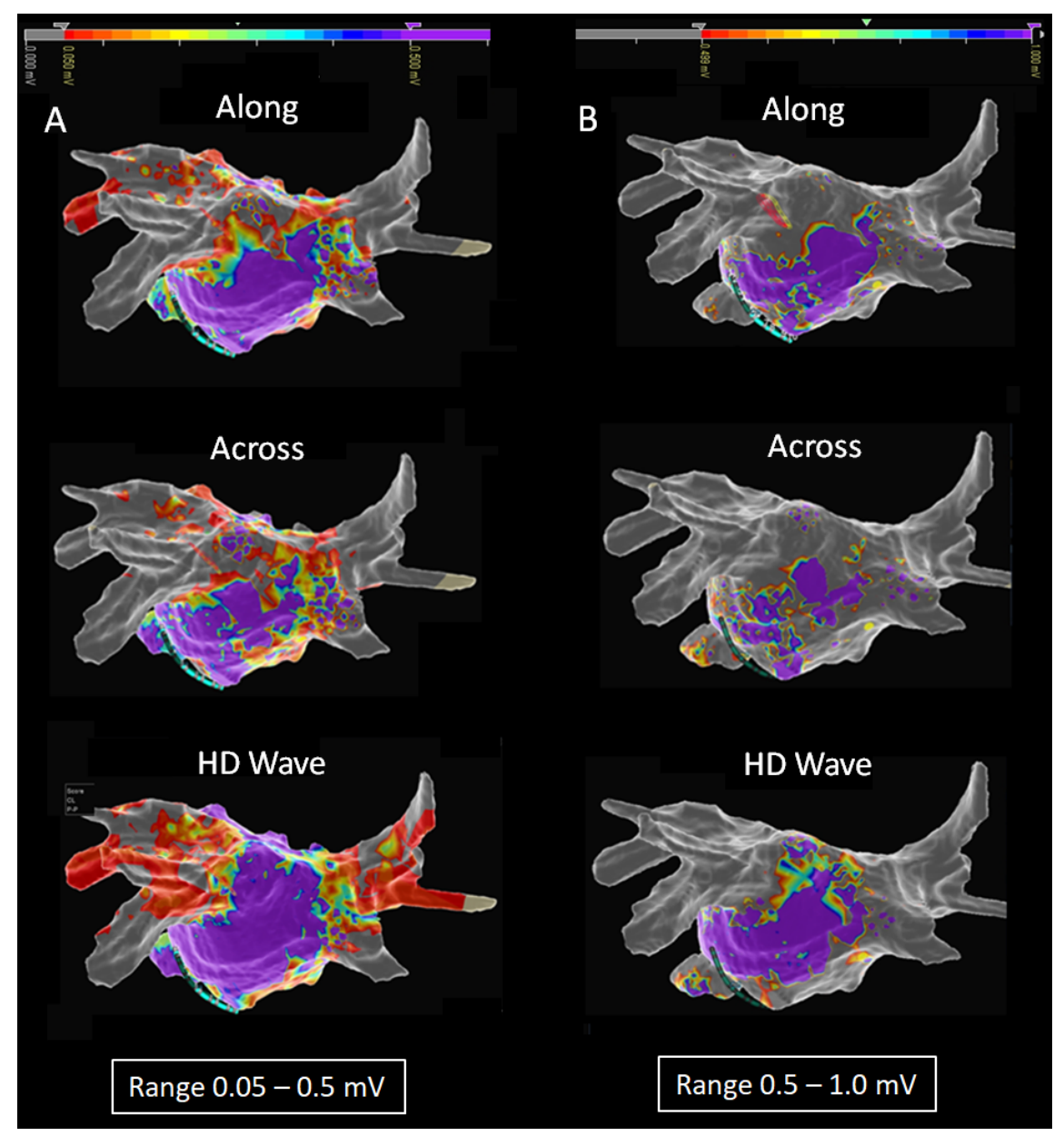

A. PA projection. Qualitative analysis after setting $0.05-0.5 \mathrm{mV}$ voltage range. Bipolar maps in along and across configurations showed LV in the PW, which we considered compatible with patchy fibrosis (set of different colors), while in the IW only normal tissue (purple color) was observed in the along configuration and partially patchy fibrosis in the across configuration. Finally, omnipolar maps in the HD-wave configuration showed normal tissue in both the PW and IW. Note the gray color in the bipolar map, which demonstrates isolation of pulmonary veins, and the red color in the omnipolar map, which seems not to completely prove PVI. B. Re-evaluation after setting 0.5-1.0 $\mathrm{mV}$ voltage range. The along and across bipolar HD maps showed an LV increase, with a shift to gray (transformation from potential patchy fibrosis into scar). The HD-wave map also showed an LV increase, but with a shift from purple to a set of different colors (transformation from normal tissue to potential patchy fibrosis). Note that pulmonary veins are isolated in both bipolar and HD-wave maps (gray color in both).

Fig. 4. Choice of the optimal range within which to identify potential patchy fibrosis 


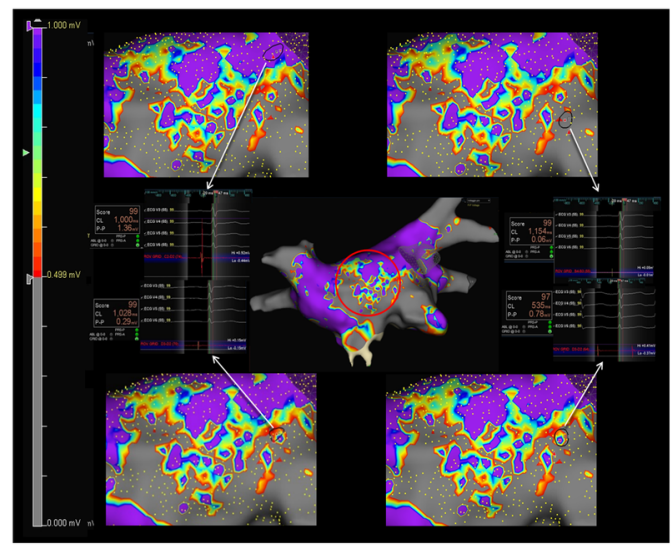

Magnification of a map acquired in the $0.5-1.0 \mathrm{mV}$ range. In this case, minimum and maximum values close to and inside the set of different colors were $0.06-1.36 \mathrm{mV}$ and $0.29-0.68 \mathrm{mV}$, respectively. These values helped to define the optimal new range of $0.3-0.6 \mathrm{mV}$ used to identify potential patchy fibrosis, and was derived from the average of all points analyzed, expressed as the mean of maximum and minimum values.

Fig. 5. MLV: comparison of Omnipolar HD-Grid versus Multipolar Pentarey catheter 


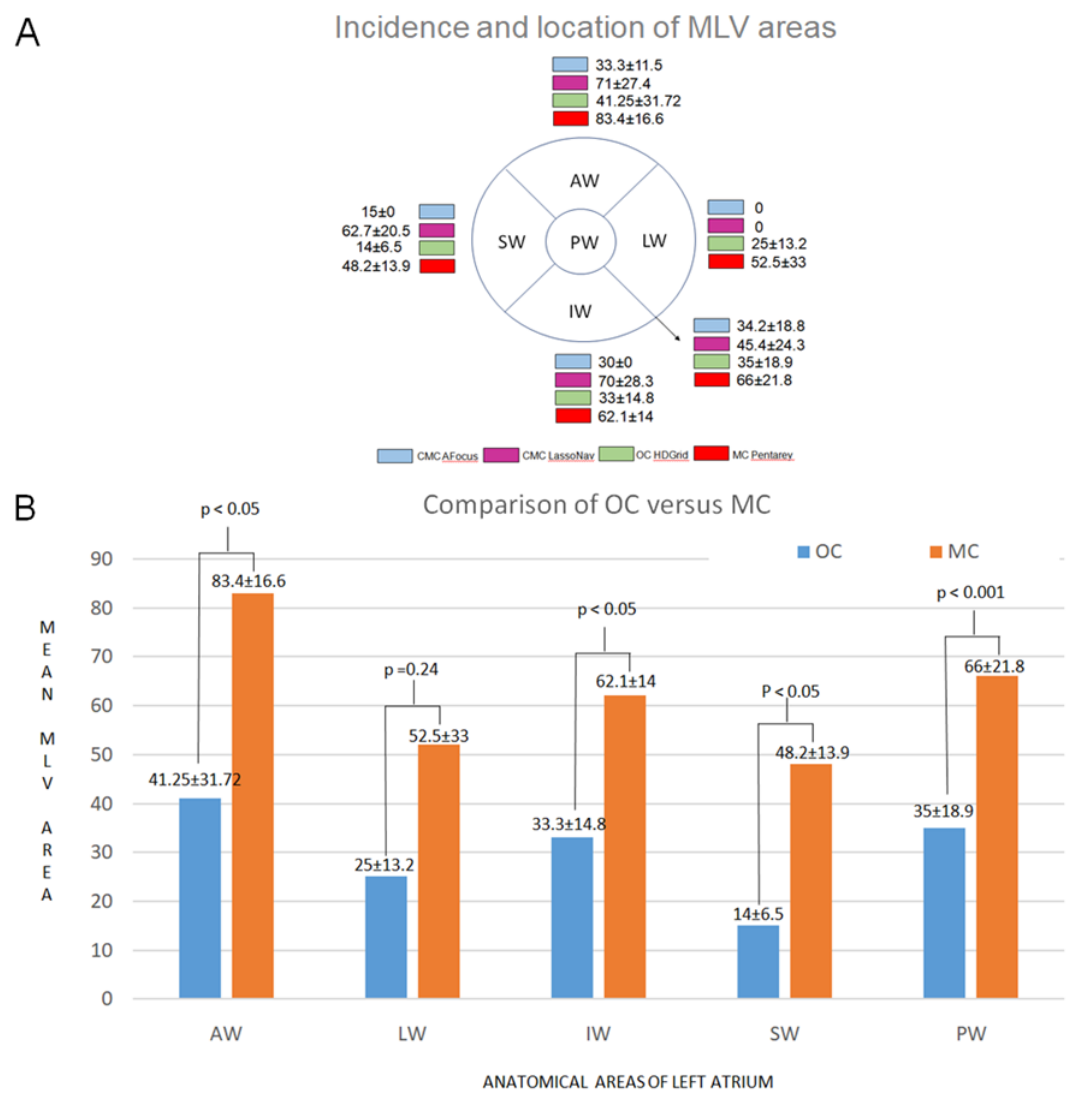

Incidence and location of Marked Low Voltage (MLV) areas acquired with Circular catheters (CMC) AFocus and LassoNav, omnipolar catheter (OC) and multipolar catheter (MC). MLVareas were quantified as a percentage of the surface of each left atrial anatomical area analyzed,and were, in turn, calculated in square centimeters through the measurements yielded by the 3D mapping system used. The values are expressed as mean \pm SD. B. Comparison between OC and MC.OC identified a significantly lower percentage of MLVthan MCin all the anatomical areas analyzed, with the exception ofthe LW. AW=anterior wall; IW=inferior wall; $L W=$ lateral wall; $P W=$ posterior wall; $S W=$ septal wallFig. 6. Kaplan-Meier estimation of the time to atrial fibrillation recurrence 

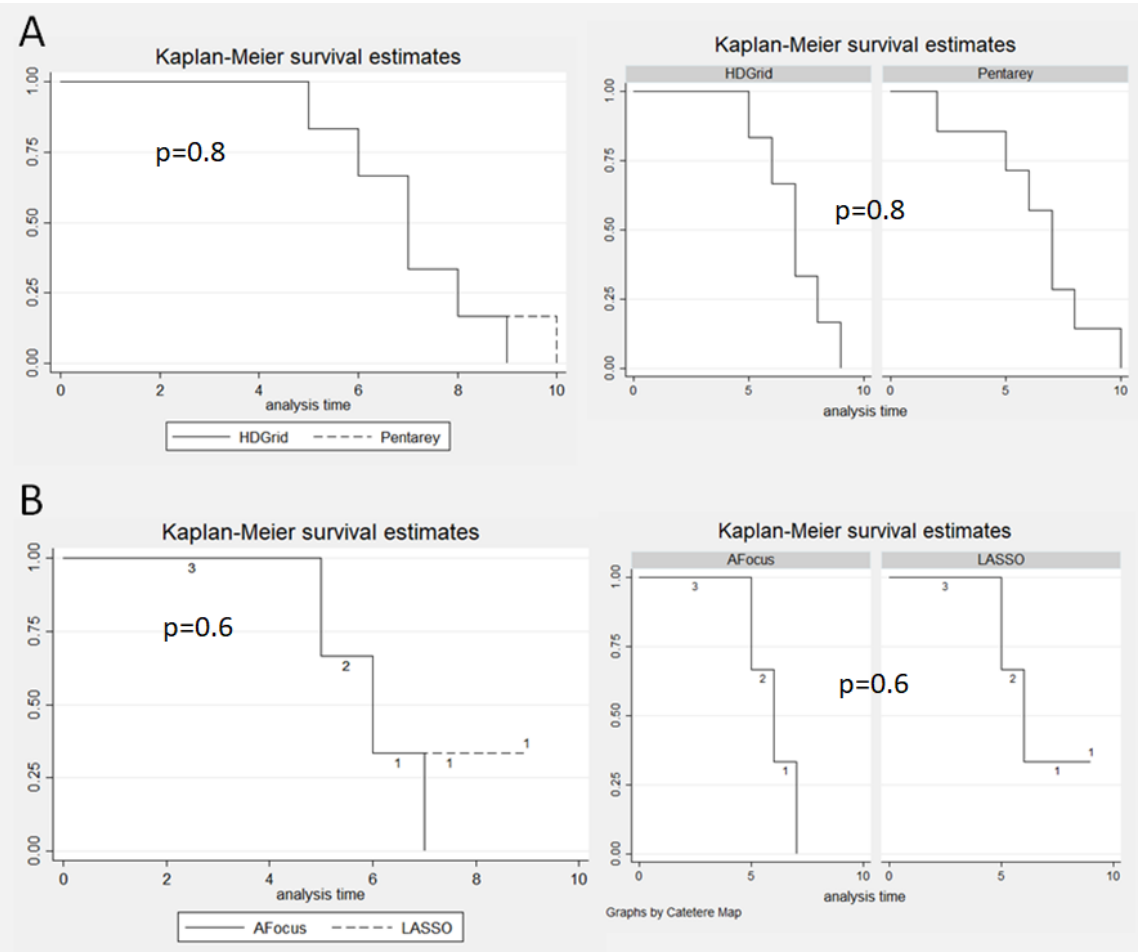

Kaplan-Meier curves showing freedom from atrial fibrillation (AF) after the first 3 months of follow-up (blanking period) in patients with HD maps acquired by means of the OC HD-Grid and MC Pentarey (panel A), and in those with HD maps acquired by means of CMC AFocus and LassoNav (panel B). Freedom from $\mathrm{AF}$ was comparable in all patients (AFocus versus LassoNav $\mathrm{p}=0.6$; OC versus $\mathrm{MC} \mathrm{p}=0.8$ ). 